\title{
Early presentation of urinary retention in multiple system atrophy: can the disease begin in the sacral spinal cord?
}

\author{
Jalesh N. Panicker ${ }^{1,2} \cdot$ Sara Simeoni ${ }^{1}(1) \cdot$ Yasuo Miki ${ }^{3,4} \cdot$ Amit Batla $^{2,5} \cdot$ Valeria lodice $^{2,6} \cdot$ Janice L. Holton $^{3}$. \\ Ryuji Sakakibara $^{7} \cdot$ Thomas T. Warner $^{3}$
}

Received: 21 August 2019 / Revised: 16 October 2019 / Accepted: 19 October 2019 / Published online: 12 November 2019

(c) The Author(s) 2019

\begin{abstract}
Lower urinary tract (LUT) dysfunction presents early in multiple system atrophy (MSA), usually initially as urinary urgency, frequency and incontinence, and voiding difficulties/urinary retention becomes apparent over time. We have observed a subset of patients who instead presented initially with urinary retention requiring catheterisation. At presentation, these patients had only subtle neurological signs that would not fulfil the diagnostic criteria of MSA; however, the anal sphincter electromyography (EMG) was abnormal and they reported bowel and sexual dysfunction, suggesting localisation at the level of the sacral spinal cord. They subsequently developed classical neurological signs, meeting the diagnostic criteria for probable MSA. One patient was confirmed to have MSA at autopsy. We postulate that in a subset of patients with MSA, the disease begins in the sacral spinal cord and then spreads to other regions resulting in the classical signs of MSA. The transmissibility of alpha-synuclein has been demonstrated in animal models and the spread of pathology from sacral cord to other regions of the central nervous system is therefore plausible. Patients presenting with urinary retention and mild neurological features would be an ideal group for experimental trials evaluating neuroprotection in MSA
\end{abstract}

Keywords MSA $\cdot$ Urinary retention $\cdot$ Multiple system atrophy $\cdot$ EMG $\cdot$ Sacral cord

Sara Simeoni

simeoni_sara85@yahoo.it

1 Department of Uro-Neurology, The National Hospital for Neurology and Neurosurgery, Queen Square, London WC1N 3BG, UK

2 UCL Queen Square Institute of Neurology, London WC1N 3BG, UK

3 Reta Lila Weston Institute of Neurological Studies and Queen Square Brain Bank, UCL Queen Square Institute of Neurology, London, UK

4 Department of Neuropathology, Institute of Brain Science, Hirosaki University Graduate School of Medicine, Hirosaki, Japan

5 Department of Neurology, Luton and Dunstable University Hospital, Luton, UK

6 Autonomics Unit, The National Hospital for Neurology and Neurosurgery, Queen Square, London WC1N 3BG, UK

7 Neurology, Internal Medicine, Sakura Medical Center, Toho University, Sakura, Japan

\section{Introduction}

Multiple system atrophy (MSA) is a progressive neurodegenerative disease characterised by autonomic dysfunction and/or parkinsonism, cerebellar or pyramidal features [1]. Pathologically, $\alpha$-synuclein-positive glial cytoplasmic inclusions (GCI) and neuronal loss are seen in the substantia nigra, caudate, putamen, globus pallidus, inferior olives, pontine nuclei, and cerebellar Purkinje cells. In the spinal cord, degeneration is evident in the intermediolateral (IML) cell columns and Onuf's nucleus [2,3].

Autonomic dysfunction presents as lower urinary tract (LUT) dysfunction, erectile dysfunction (ED) and orthostatic hypotension $(\mathrm{OH})$. LUT symptoms present on an average 2.8 years sooner than motor symptoms and may be the initial presenting complaint in nearly $20 \%$ of patients [4], preceding $\mathrm{OH}[5,6]$. Commonly, patients present initially with storage LUT symptoms such as nocturia, urgency and urinary incontinence, and over time voiding difficulties become more apparent [5]. The post-void residual (PVR) volume increases with disease progression, and in a published series the mean PVR in the first year of disease was 
$71 \mathrm{~mL}$, which increased to $170 \mathrm{~mL}$ by the fifth year. Urinary retention requiring catheterisation is rarely reported at disease onset, and increases to $14 \%$ over a 5 -year period [7].

However, we have observed and we illustrate in this paper a subset of representative patients who initially presented with severe urinary retention requiring catheterisation, bowel and sexual dysfunction, and abnormal anal sphincter electromyography (EMG) which is otherwise characteristic of sacral spinal cord (SSC) lesions, and only subtle neurological signs that would not fulfil the diagnostic criteria of MSA. Subsequently, the disease progressed and all of them developed characteristic signs of MSA leading to clinical diagnosis.

We postulate that at least in a subset of patients, the pathology of MSA can start in the sacral spinal cord and subsequently spread to other areas of the central nervous system.

Table 1 summarises the findings of six cases and a representative case is given below.

\section{Patient 1}

Patient 1 developed insidious onset voiding difficulties, urinary frequency and urgency, and when assessed 2 years later was found to be in urinary retention. He was experiencing erectile and ejaculatory dysfunction, and constipation. At the time of referral for investigating the cause for urinary retention, he was unable to void naturally and was performing intermittent self-catheterisation four times a day. The neurological examination revealed brisk deep tendon reflexes and no other neurological signs. The blood pressure was stable. MR imaging did not show any significant atrophy in the brain, or compressive lesions of the conus or cauda equina. The results of anal sphincter EMG and urodynamics testing are presented in Fig. 1.

Two years later he developed postural instability, a cerebellar gait, dysphagia and REM sleep behaviour disorder (RBD). Autonomic testing showed evidence for cardiovascular autonomic failure and a diagnosis of probable MSA was made. The following year, he developed dysarthria, bradykinesia, rigidity and worsening cerebellar signs and became wheelchair bound. Repeat brain MRI showed mild parenchymal volume loss within the posterior fossa, mainly affecting the middle cerebellar peduncles, although the cerebellar fissures also appeared marginally more prominent. The pons also appeared to have lost volume, with the impression of more conspicuous midline linear T2-hyperintense signal change. The basal ganglia structures returned normal signal.

His mobility deteriorated and he became bed bound and required a urethral indwelling catheter. He died of complications related to MSA at the age of 43. At autopsy, histological examination confirmed the diagnosis of MSA with neuronal loss predominantly affecting

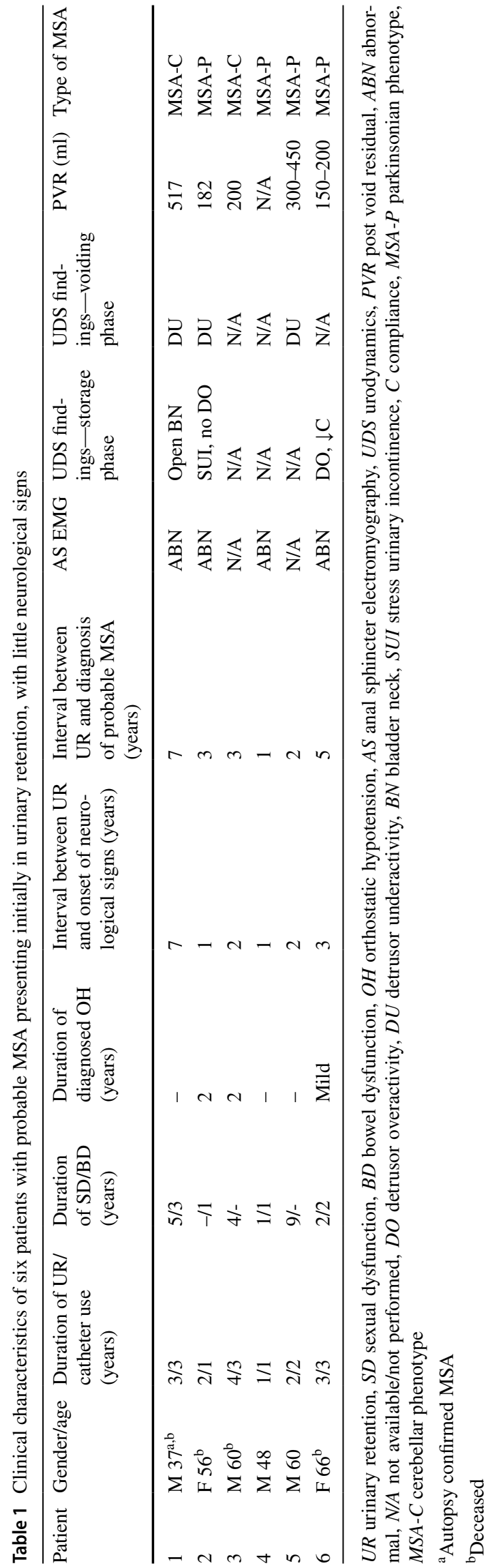




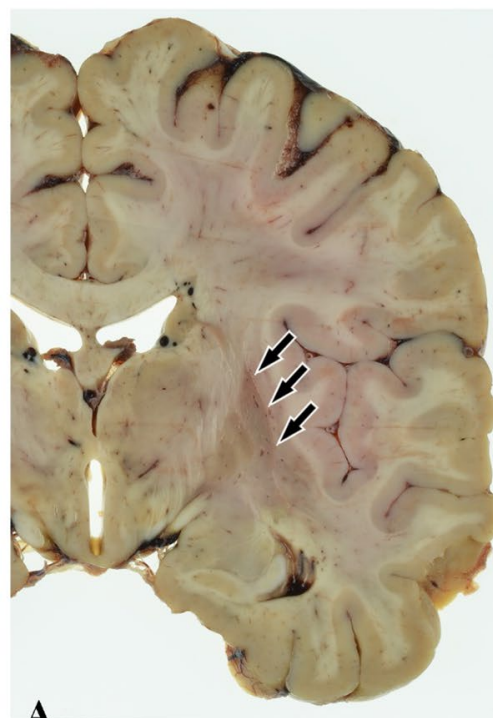

$\mathbf{A}$

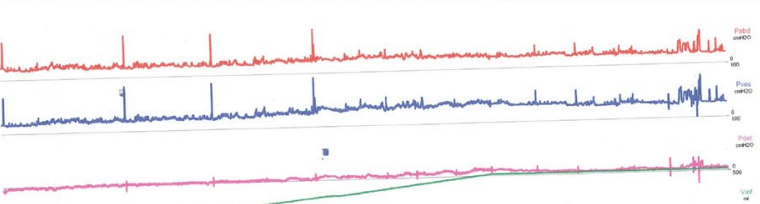

F

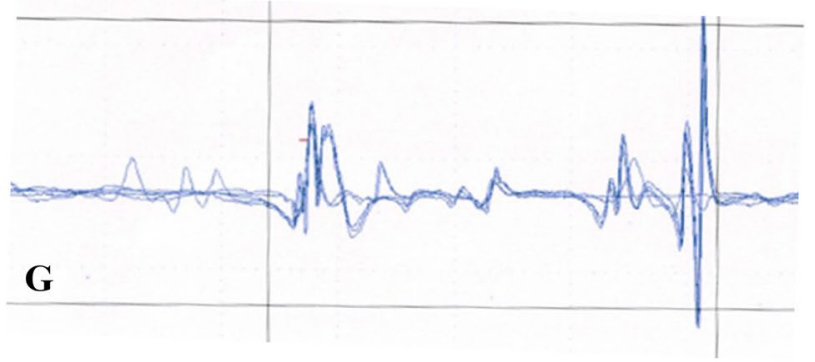

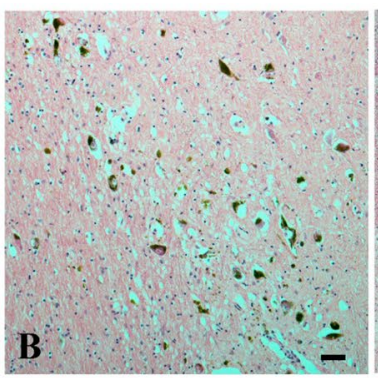

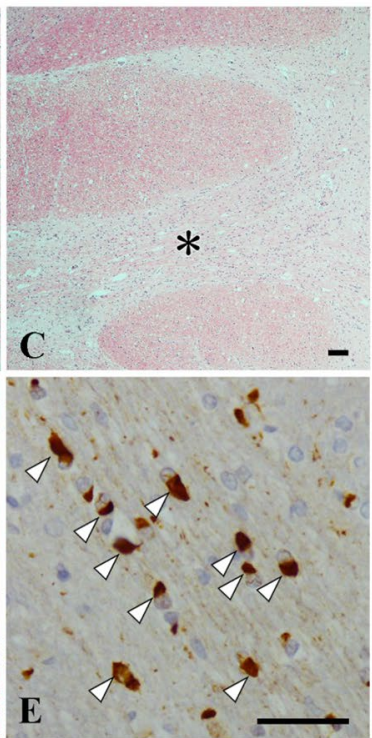

D

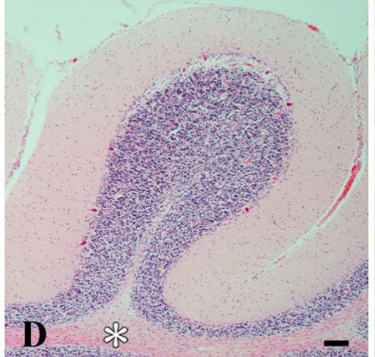

Fig. 1 Findings of case 1. a-e Pathological findings. Coronal section of the left cerebral hemisphere showing atrophy and dark discolouration in the putamen (arrows) (a). Moderate loss of dopaminergic neurons in the substantia nigra (b). Moderate depletion of neurons with rarefaction of the transvers fibres in the pons (black asterisk) (c). Moderate loss of Purkinje cells along with loss of myelinated fibres (white asterisk) (d). Glial cytoplasmic inclusions immunopositive for $\alpha$-synuclein (arrowheads) (e). b-d Haematoxylin-eosin staining, e $\alpha$-synuclein immunohistochemistry. Bars $=50 \mu \mathrm{m}(\mathbf{b}, \mathbf{e}) ; 100 \mu \mathrm{m}$ (c, d). f Urodynamics findings showing normal compliance, normal

the olivopontocerebellar structures, and to a lesser extent the striatonigral structures, corresponding to the OPCA subtype. There were very frequent glial cytoplasmic inclusions and threads in the pontine base with slightly less frequent deposits in the tegmentum, including the locus coeruleus (Fig. 1). bladder capacity, stable detrusor during fill, normal bladder sensation during fill, and acontractile detrusor during the voiding phase. Pabd intra-abdominal pressure, Pves intravesical pressure, Pdet detrusor pressure, Vinf infused volume, Qura urine flow. g Concentric needle EMG of the external anal sphincter. Duration of the recorded motor unit is $38.54 \mathrm{~ms}$, which is prolonged and suggests chronic reinnervation. The mean duration of MUPs during the study was $19 \mathrm{~ms}$ (normal $<10 \mathrm{~ms}$ ) and the EMG was compatible with a diagnosis of multiple system atrophy (gain $0.2 \mathrm{mV} /$ division, sweep speed $10 \mathrm{~ms} /$ division)

\section{Discussion}

In this series of six cases, the initial clinical picture was dominated by urinary retention requiring either intermittent or an indwelling catheter, accompanied by bowel and sexual dysfunction with only subtle neurological signs. 
Patients subsequently developed neurological signs and only at this point met the diagnostic criteria for probable MSA [1]. Brain tissue from patient 1 clearly demonstrated a pattern of neuronal loss and the presence of glial cytoplasmic inclusions and threads consistent with the diagnosis of MSA.

This presentation is in contrast to the more common clinical presentation of urinary retention developing after the onset of other neurological signs, usually after the second year of illness [7]. Urodynamic testing revealed detrusor underactivity which, in the setting of neurological disease, occurs as a result of a lesion of the sacral spinal cord or infrasacrally [8]. In MSA, urinary retention can occur due to detrusor underactivity, which has been reported in $71 \%$ of women and $63 \%$ of men in a series of patients undergoing urodynamics testing [9]. The prevalence of detrusor underactivity increases with advancing disease, paralleling worsening urinary retention over time [7], and is likely to reflect degeneration in the sacral spinal cord, specifically the parasympathetic IML column.

Urinary retention may also result from involvement of the brainstem such as the pontine micturition centre [1013]. It was notable that patients in this series had very little neurological symptoms suggesting brainstem dysfunction at the time of presentation with urinary retention. Incomplete bladder emptying in MSA may also occur due to detrusor sphincter dyssynergia, reflecting suprasacral spinal cord involvement $[7,8]$.

Concentric needle EMG of the anal sphincter revealed abnormal reinnervated motor unit potentials in all the patients who were tested. Nerve fibres innervating the striated anal sphincter are derived from the Onuf's nucleus in the ventral anterior horn of the sacral spinal cord primarily at the $\mathrm{S} 2$ level, but extending between $\mathrm{S} 1$ and $\mathrm{S} 3$ segments [14]. An abnormal EMG suggests a lesion affecting the sacral somatic motor efferent pathway including the Onuf's nucleus [15-17].

Co-existent sexual and bowel complaints, and the findings of an abnormal anal sphincter EMG, would suggest a neurological cause for urinary retention with likely localisation at the sacral spinal cord or nerve roots $[8,18]$. Orthostatic hypotension was present in some of the patients at an early stage, and a peripheral autonomic disorder such as pure autonomic failure (PAF) may have been considered in the differential diagnosis; however, the anal sphincter EMG is reported to be normal in this condition $[15,19]$. However the patients in this series subsequently developed classical extrapyramidal and cerebellar involvement and a diagnosis of probable MSA was made.

We speculate that a subset of patients with MSA present with urinary retention and few neurological signs and the pathology in these cases begins in the sacral cord. The lower spinal cord is particularly involved in MSA and pathological studies reveal neuronal loss and gliosis specifically affecting the IML cell columns, anterior horn cells, pyramidal tracts, and Onuf's nucleus [20, 21]. Moreover, Schwann cells of the anterior nerves of the sacral cord may show accumulation of phosphorylated $\alpha$-synuclein [22]. In proteolipid protein- $\alpha$-synuclein transgenic mice demonstrating MSA-like $\alpha$-synuclein inclusions and parkinsonian motor deficits, neuronal loss and $\alpha$-synuclein overexpression have been demonstrated in the Onuf's nucleus analogue as early as 2 months of life [23]. Neurons of the Onuf's nucleus share features between somatic and autonomic neurons and is susceptible to neurodegenerative conditions affecting the autonomic nervous system such a MSA [21, 24]. Preganglionic neurons of the IML column of the sacral spinal cord are responsible for the innervation of the detrusor [25] and involvement of this region is likely to be responsible for detrusor failure. Routine MRI sequences of the lower cord are insensitive to identify degenerative changes in the lumbosacral spinal cord; however, high-resolution MR imaging protocols have successfully imaged the lumbosacral enlargement [26] and conus medullaris [27] and changes of grey matter atrophy have been demonstrated in MSA [28].

The transmissibility of $\alpha$-synuclein has been demonstrated in animal models following inoculation of tissue from MSA patients [29], and the transmission of pathology from sacral cord to other regions of the central nervous system is therefore plausible [30]. Other neurological signs develop with progressive involvement of the brain stem, cerebellum and basal ganglia. Further studies are required to explore this pathological progression from spinal cord to brain, as well as to prospectively chart the clinical progression of patients with suspected MSA who present initially with voiding dysfunction.

In conclusion, a subset of patients with probable MSA may present initially with urinary retention. The findings of detrusor underactivity in urodynamic testing and findings of reinnervation in the anal sphincter EMG suggests possible involvement of the sacral spinal cord. A limitation to the study is that spinal cord tissue was not analysed; however as tissue diagnosis was made at end stage, the inclusion of sacral cord pathological findings would not help support the hypothesis that the disease process actually begans in the spinal cord. Nevertheless, considering the degree of spinal cord involvement in this condition, both brain and spinal cord tissue should be analysed in future pathological studies of MSA. If further studies support the progression of pathology from sacral cord to brain in early MSA, patients presenting with urinary retention and mild neurological features would be an ideal group for experimental trials evaluating neuroprotection in MSA.

Acknowledgements JNP undertook this work at UCLH/UCL Institute of Neurology and was supported in part by funding from the United 
Kingdom's Department of Health NIHR Biomedical Research Centres funding scheme. YM was supported by the Uehara Memorial Foundation. JLH was supported by the Multiple System Atrophy Trust; the Multiple System Atrophy Coalition; Fund Sophia, managed by the King Baudouin Foundation and Karin \& Sten Mortstedt CBD Solutions. Queen Square Brain Bank is supported by the Reta Lila Weston Institute for Neurological Studies and the Medical Research Council UK.

\section{Compliance with ethical standards}

Conflicts of interest The authors declare that they have no conflict of interest.

Ethics standards All procedures performed in this study were in accordance with the ethical standards of the institutional and national research committee and with the 1964 Helsinki declaration and its later amendments or comparable ethical standards.

Informed consent For this type of study formal consent is not required.

Open Access This article is distributed under the terms of the Creative Commons Attribution 4.0 International License (http://creativeco mmons.org/licenses/by/4.0/), which permits unrestricted use, distribution, and reproduction in any medium, provided you give appropriate credit to the original author(s) and the source, provide a link to the Creative Commons license, and indicate if changes were made.

\section{References}

1. Gilman S, Wenning GK, Low PA, Brooks DJ, Mathias CJ, Trojanowski JQ, Wood NW, Colosimo C, Durr A, Fowler CJ, Kaufmann H, Klockgether T, Lees A, Poewe W, Quinn N, Revesz T, Robertson D, Sandroni P, Seppi K, Vidailhet M (2008) Second consensus statement on the diagnosis of multiple system atrophy. Neurology 71:670-676

2. Quinn N (1989) Multiple system atrophy - the nature of the beast. J Neurol Neurosurg Psychiatry 52(Suppl):78-89

3. Lantos PL, Papp MI (1994) Cellular pathology of multiple system atrophy: a review. J Neurol Neurosurg Psychiatry 57:129-133

4. Sakakibara R, Panicker J, Simeoni S, Uchiyama T, Yamamoto T, Tateno F, Kishi M, Aiba Y (2018) Bladder dysfunction as the initial presentation of multiple system atrophy: a prospective cohort study. Clin Auton Res. https://doi.org/10.1007/s1028 6-018-0550-y

5. Sakakibara R, Hattori T, Uchiyama T, Kita K, Asahina M, Suzuki A, Yamanishi T (2000) Urinary dysfunction and orthostatic hypotension in multiple system atrophy: which is the more common and earlier manifestation? J Neurol Neurosurg Psychiatry 68:65-69

6. Kirchhof K, Apostolidis AN, Mathias CJ, Fowler CJ (2003) Erectile and urinary dysfunction may be the presenting features in patients with multiple system atrophy: a retrospective study. Int J Impot Res 15:293-298

7. Ito T, Sakakibara R, Yasuda K, Yamamoto T, Uchiyama T, Liu Z, Yamanishi T, Awa Y, Yamamoto K, Hattori T (2006) Incomplete emptying and urinary retention in multiple-system atrophy: when does it occur and how do we manage it? Mov Disord 21:816-823

8. Panicker JN, Fowler CJ, Kessler TM (2015) Lower urinary tract dysfunction in the neurological patient: clinical assessment and management. Lancet Neurol 14:720-732
9. Sakakibara R, Hattori T, Uchiyama T, Yamanishi T (2001) Videourodynamic and sphincter motor unit potential analyses in Parkinson's disease and multiple system atrophy. J Neurol Neurosurg Psychiatry 71:600-606

10. Kirby R, Fowler C, Gosling J, Bannister R (1986) Urethro-vesical dysfunction in progressive autonomic failure with multiple system atrophy. J Neurol Neurosurg Psychiatry 49:554-562

11. Sakakibara R, Hattori T, Yasuda K, Yamanishi T (1996) Micturitional disturbance and the pontine tegmental lesion: urodynamic and MRI analyses of vascular cases. J Neurol Sci 141:105-110

12. Sakakibara R, Hattori T, Fukutake T, Mori M, Yamanishi T, Yasuda K (1998) Micturitional disturbance in herpetic brainstem encephalitis; contribution of the pontine micturition centre. J Neurol Neurosurg Psychiatry 64:269-272

13. Komiyama A, Kubota A, Hidai H (1998) Urinary retention associated with a unilateral lesion in the dorsolateral tegmentum of the rostral pons. J Neurol Neurosurg Psychiatry 65:953-954

14. Panicker JN (2019) The Onuf's nucleus, serotonin and spinal cord injury. BJU Int 123:716-717

15. Sakakibara R, Uchiyama T, Yamanishi T, Kishi M (2009) Sphincter EMG as a diagnostic tool in autonomic disorders. Clin Auton Res 19:20-31

16. Palace J, Chandiramani VA, Fowler CJ (1997) Value of sphincter electromyography in the diagnosis of multiple system atrophy. Muscle Nerve 20:1396-1403

17. Podnar S, Fowler CJ (2004) Sphincter electromyography in diagnosis of multiple system atrophy: technical issues. Muscle Nerve 29:151-156

18. Smith MD, Seth JH, Fowler CJ, Miller RF, Panicker JN (2013) Urinary retention for the neurologist. Pract Neurol 13:288-291

19. Sakakibara R, Hattori T, Uchiyama T, Asahina M, Yamanishi T (2000) Micturitional disturbance in pure autonomic failure. Neurology 54:499-501

20. Wenning GK, Tison F, Ben Shlomo Y, Daniel SE, Quinn NP (1997) Multiple system atrophy: a review of 203 pathologically proven cases. Mov Disord 12:133-147

21. Konno H, Yamamoto T, Iwasaki Y, Iizuka H (1986) Shy-Drager syndrome and amyotrophic lateral sclerosis. Cytoarchitectonic and morphometric studies of sacral autonomic neurons. J Neurol Sci 73:193-204

22. Nakamura K, Mori F, Kon T, Tanji K, Miki Y, Tomiyama M, Kurotaki H, Toyoshima Y, Kakita A, Takahashi H, Yamada M, Wakabayashi K (2015) Filamentous aggregations of phosphorylated $\alpha$-synuclein in Schwann cells (Schwann cell cytoplasmic inclusions) in multiple system atrophy. Acta Neuropathol Commun. 21:3-29

23. Stefanova N, Reindl M, Neumann M, Haass C, Poewe W, Kahle PJ, Wenning GK (2005) Oxidative stress in transgenic mice with oligodendroglial alpha-synuclein overexpression replicates the characteristic neuropathology of multiple system atrophy. Am J Pathol 166:869-876

24. Mannen T, Iwata M, Toyokura Y, Nagashima K (1982) The Onuf's nucleus and the external anal sphincter muscles in amyotrophic lateral sclerosis and Shy-Drager syndrome. Acta Neuropathol $58: 255-260$

25. Fowler CJ, Griffiths D, de Groat WC (2008) The neural control of micturition. Nat Rev Neurosci 9:453-466

26. Ugorji CO, Samson RS, Liechti MD, Panicker JN, Miller DH, Wheeler-Kingshott CA, Yiannakas MC (2015) Grey and white matter magnetisation transfer ratio measurements in the lumbosacral enlargement: a pilot in vivo study at 3T. PLoS ONE ONE 10:e0134495

27. Yiannakas MC, Liechti MD, Budtarad N, Cullinane P, Yang X, Toosy AT, Panicker JN, Gandini Wheeler-Kingshott CAM (2018) Gray vs. white matter segmentation of the conus medullaris: 
reliability and variability in healthy volunteers. J Neuroimaging 29(3):410-417

28. Liechti MD, Yiannakas M, Budtarad N, Toosy AT, Yang X, Prados F, Miller D, Houlden H, Gandini Wheeler-Kingshott C, Panicker JN (2017) Assessing changes within the lumbosacral spinal cord in neurological disease: preliminary results of a pilot in vivo MRI study. In: International society for magnetic resonance in medicine 25 th annual meeting \& exhibition, vol 25

29. Watts JC, Giles K, Oehler A, Middleton L, Dexter DT, Gentleman SM, DeArmond SJ, Prusiner SB (2013) Transmission of multiple system atrophy prions to transgenic mice. Proc Natl Acad Sci USA 110:19555-19560

30. Prusiner SB, Woerman AL, Mordes DA, Watts JC, Rampersaud R, Berry DB, Patel S, Oehler A, Lowe JK, Kravitz SN, Geschwind DH, Glidden DV, Halliday GM, Middleton LT, Gentleman SM, Grinberg LT, Giles K (2015) Evidence for alpha-synuclein prions causing multiple system atrophy in humans with parkinsonism. Proc Natl Acad Sci USA 112:E5308-5317 